\title{
Operation of high-temperature superconductor magnetometer with submicrometer bicrystal junctions
}

\author{
M. I. Faley, ${ }^{\text {a) }}$ U. Poppe, and K. Urban \\ Institut für Festkörperforschung, Forschungszentrum Jülich GmbH, D-52425 Jülich, Germany
}

V. Yu. Slobodchikov and Yu. V. Maslennikov

Institute of Radio Engineering and Electronics, 101999 Moscow, Russia

A. Gapelyuk, B. Sawitzki, and A. Schirdewan
Franz Volhard Hospital, D-13125 Berlin, Germany

(Received 6 June 2002; accepted for publication 29 July 2002)

We investigated the noise properties of dc superconducting quantum interference device flip-chip magnetometers with submicrometer-wide bicrystal junctions operating at $77.4 \mathrm{~K}$. The noise of the magnetometers with electronics was about $6 \mathrm{fT} / \sqrt{ } \mathrm{Hz}$ at frequencies above $100 \mathrm{~Hz}$ increasing up to about $20 \mathrm{fT} / \sqrt{ } \mathrm{Hz}$ at $1 \mathrm{~Hz}$. The operation of the magnetometers was characterized in an electronic axial first order gradiometer system, which was employed for biomagnetic measurements. The system demonstrated a gradient resolution of about $1 \mathrm{fT} / \mathrm{cm} \sqrt{ } \mathrm{Hz}$ at $77.4 \mathrm{~K}$ and stable operation in a standard magnetically shielded room under clinical conditions. (C) 2002 American Institute of Physics. [DOI: 10.1063/1.1508419]

At the present stage of the development of high temperature superconductor (HTS) technology a production of HTS dc superconducting quantum interference device (SQUID) magnetometers with a magnetic field resolution below $10 \mathrm{fT} /{ } \mathrm{Hz}$ at $77.4 \mathrm{~K}$ is possible. ${ }^{1-3} \mathrm{~A}$ further improvement of the resolution and yield of the sensors is required for the development of multichannel systems. The sensitivity of dcSQUIDs can be improved with Josephson junctions having a higher normal state resistance $R_{n}$ and characteristic voltage $V_{c}=I_{c} R_{n}$, where $I_{c}$ is the critical current of the junction. It was observed ${ }^{4,5}$ that the $I_{c} R_{n}$ product of bicrystal junctions increases with critical current density $J_{c}$ as $I_{c} R_{n} \propto\left(J_{c}\right)^{0.6}$. In order to keep $I_{c}$ fixed at an optimal level for SQUID operation, to provide narrow junction conditions, and simultaneously to increase $J_{c}$ and $R_{n}$ it is necessary to reduce the junction width to a submicrometer scale. It is very important to prove the quality and applicability of such submicrometer junctions for HTS dc-SQUID magnetometers operating at $77.4 \mathrm{~K}$.

The development, optimization, and demonstration of HTS structures are very effective by incorporating them in a system for some particular sophisticated application. Biomagnetic measurements are especially interesting because they require an ultimate combination of field and spatial resolution achievable exclusively with SQUID sensors. A flip-chip arrangement of the SQUID with a multiturn flux transformer allows to obtain the magnetometers with the highest resolution, which is partially limited by the noise of the junctions. Here we present the characteristics of HTS dc-SQUID flipchip magnetometers with submicrometer-wide bicrystal junctions and their operation in a magnetocardiography (MCG) system under clinical conditions at $77.4 \mathrm{~K}$.

The high-oxygen-pressure dc-sputtering technique ${ }^{6}$ was used for the deposition of the $\mathrm{YBa}_{2} \mathrm{Cu}_{3} \mathrm{O}_{7-x}$ films on $24^{\circ}$

${ }^{a)}$ Electronic mail: m.faley@fz-juelich.de bicrystal $\mathrm{SrTiO}_{3}$ substrates. C-oriented 200-nm-thick $\mathrm{YBa}_{2} \mathrm{Cu}_{3} \mathrm{O}_{7-x}$ films show typical critical current densities $J_{c}$ of about $6 \times 10^{6} \mathrm{~A} / \mathrm{cm}^{2}$ at $77.4 \mathrm{~K}$ and a transition temperature $T_{c}$ above $91 \mathrm{~K}$ measured by magnetic susceptibility. Some inclusions of secondary phases served as effective pinning centers. The junctions (see Fig. 1) had a width between 0.4 and $1 \mu \mathrm{m}$, a normal state resistance of about $10 \Omega$, and a critical current density of about $2 \times 10^{4} \mathrm{~A} / \mathrm{cm}^{2}$ at $77.4 \mathrm{~K}$. The junctions were prepared by optimized conventional photolithography with AZ5214 photoresist, $0.3 \mu \mathrm{m}$ UV-light exposure, and Ar ion milling.

$16 \mathrm{~mm}$ flux transformers ${ }^{3}$ were prepared by a poly(methylmethacrylate)-photoresist technique described in Ref. 7 with a $\mathrm{PrBa}_{2} \mathrm{Cu}_{3} \mathrm{O}_{7-x}$ insulation layer between the

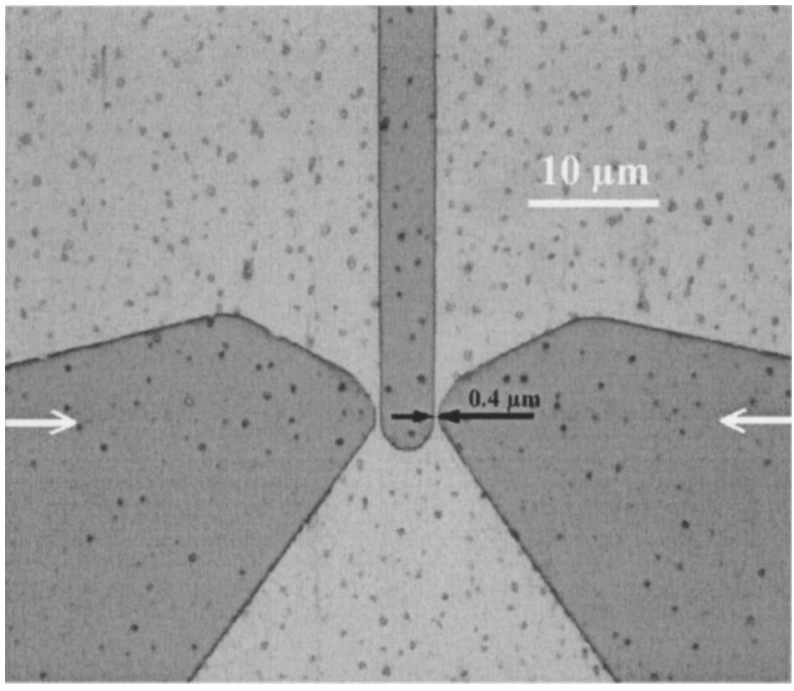

FIG. 1. Optical image of the bicrystal junctions, obtained in a combination of transmission and reflective illumination. A magnification of $\times 1000$ and a green filter were used. The white arrows indicate the position of the bicrystal boundary. The black arrows determine the width of the $0.4 \mu \mathrm{m}$ wide junction. 


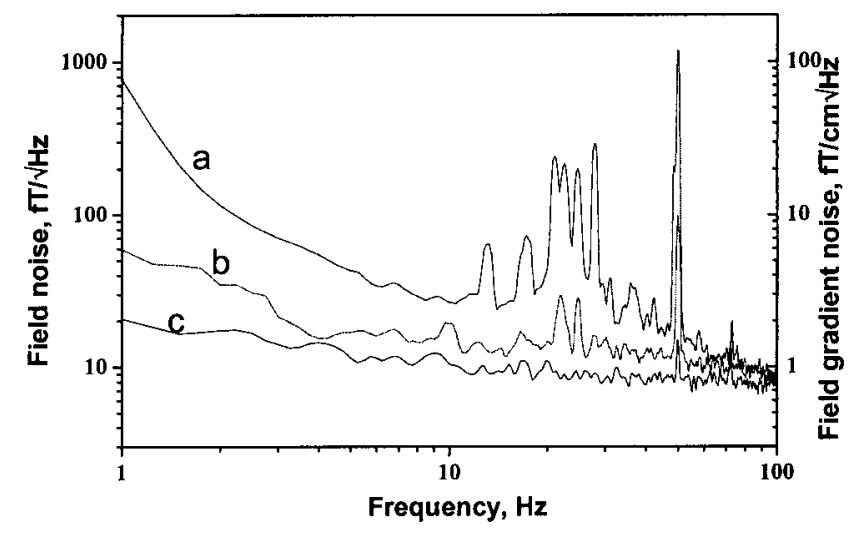

FIG. 2. Noise spectrum of single magnetometer (a) and the spectrum of the differential signal (b) measured in the MSR of the Franz Volhard Hospital at $77.4 \mathrm{~K}$. For comparison the noise of the bottom magnetometer measured in a HTS shield is included (c).

windings of the multiturn input coil and the return strip. The $\mathrm{PrBa}_{2} \mathrm{Cu}_{3} \mathrm{O}_{7-x}$ film prevents a superconducting shortage and provides a normal conducting shunt of the $16 \mathrm{~mm}$ multilayer flux transformer to avoid high-frequency resonances in the multiturn coil structure (see, e.g., Ref. 2, and references therein). The effective SQUID inductance of the flip-chip sensor was about $100 \mathrm{pT}$ and demonstrated a modulation voltage $V_{p p}$ up to $80 \mu \mathrm{V}\left(\partial V / \partial \Phi \approx \pi V_{p p} \approx 250 \mu V / \Phi_{0}\right)$ at $77.4 \mathrm{~K}$. Here $\Phi$ is the magnetic flux through the SQUID loop and $\Phi_{0}=2.05 \times 10^{-15} \mathrm{~T} / \mathrm{m}^{2}$ is the flux quantum. For comparison, similar magnetometers with $1.5 \mu \mathrm{m}$ wide junctions showed values of $V_{p p}$ of only up to about $30 \mu \mathrm{V}$ at $77.4 \mathrm{~K}$. The flip-chip magnetometers were sealed vacuum tight in fiberglass epoxy encapsulations. ${ }^{3}$ A Pt heater integrated into the encapsulation allowed trapped flux to be removed. The encapsulations damp the thermal fluctuations and provide long-time stability of the sensors.

Two encapsulated magnetometers were fixed parallel to each other on a fiberglass epoxy rod. The gradiometer baseline of $10 \mathrm{~cm}$ is determined by the distance between the flux transformers of the magnetometers. Two channel ac-bias electronics (Cryoton) provided a simultaneous operation of the magnetometers and an electronic subtraction of the output signals. The sensors were immersed in liquid nitrogen in a 1.51 fiberglass epoxy cryostat. The cryogen hold time of the cryostat with the gradiometer insert was 10 days. The cold-warm distance at the cryostat bottom was about $10 \mathrm{~mm}$.

The measurements were performed in the magnetically shielded room (MSR) of the Franz Volhard Hospital, Berlin. The current shielding factor of the MSR is about $10^{4}$ at 50 $\mathrm{Hz}$ and about 10 at $0.1 \mathrm{~Hz}$. A slow variation of the magnetic field in the middle of the MSR was observed during the daytime. A typical time constant of this drift was about $10 \mathrm{~s}$ and the amplitude was up to $5 \mathrm{nT}$. No active compensation of this low-frequency environmental field noise in the MSR was performed during the measurements. The feedback of the electronics was adjusted to follow the maximal changes of the magnetic field in the MSR.

The system noise spectrum with one of the individual magnetometers [curve (a)] and the spectrum of the differential signal [curve (b)] are presented in Fig. 2. The observed increase of the field noise at $1 \mathrm{~Hz}$ is associated with the reduced shielding factor of the MSR at low frequencies. The Downloaded 15 Dec 2006 to 134.94.122.39. Redistribution subjec

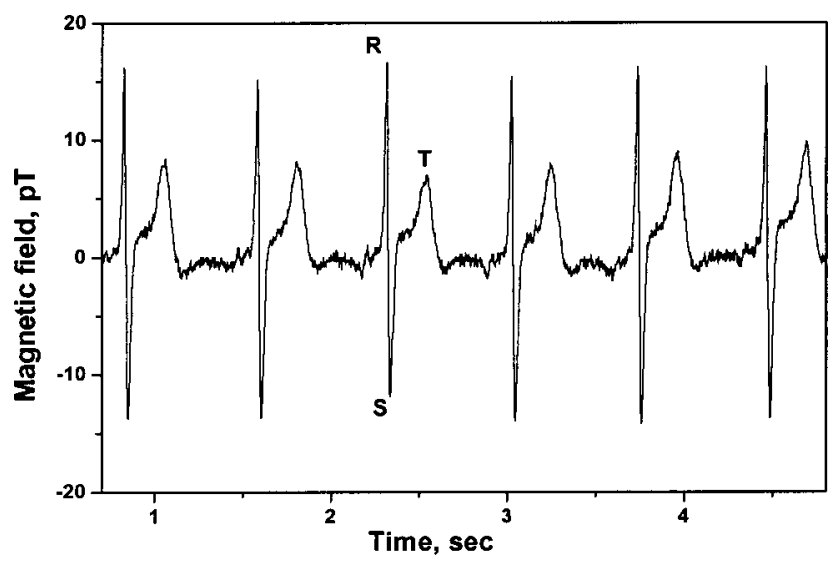

FIG. 3. Real-time MCG response of the electronic gradiometer. The frequency bandwidth ranges from 0.03 to $125 \mathrm{~Hz}$.

magnetic field signals observed in the frequency range from 10 to $30 \mathrm{~Hz}$ [curve (a)] were mainly caused by building vibrations. In a HTS shield the individual magnetometers demonstrated a field noise below $10 \mathrm{fT} / \sqrt{ } \mathrm{Hz}$ down to $10 \mathrm{~Hz}$ increasing up to $20 \mathrm{fT} / \sqrt{ } \mathrm{Hz}$ at $1 \mathrm{~Hz}$ [curve (c)].

In the differential signal an essential part of the noise determined by the low frequency signal, vibrations effects, and $50 \mathrm{~Hz}$ interference is subtracted. Taking into account the $10 \mathrm{~cm}$ baseline of the electronic gradiometer the spectrum of the differential signal is presented in Fig. 2 with the calibration scale on the right in $(\mathrm{fT} / \mathrm{cm} \sqrt{ } \mathrm{Hz})$ units. The resolution of the gradiometric signal is below $1 \mathrm{fT} / \mathrm{cm}_{\sqrt{ }} \mathrm{Hz}$ at frequencies above $100 \mathrm{~Hz}$ and below $2 \mathrm{fT} / \mathrm{cm} \sqrt{ } \mathrm{Hz}$ in the frequency range down to about $4 \mathrm{~Hz}$. No $50 \mathrm{~Hz}$ filter was applied during the measurements presented in Fig. 2. At lower frequencies there is a significant increase of the noise due to the uncompensated part of the magnetic field drift in the MSR. An active compensation of the environmental field in the MSR would improve the low-frequency resolution of the system. The white noise of the electronic gradiometer exceeded the noise of the individual magnetometers by a factor of about $\sqrt{2}$ in accordance with theoretical expectations.

The MCG measurements were performed in the MSR with a volunteer having a maximal peak-to-peak amplitude of about $30 \mathrm{pT}$ for the magnetic signal of the heart. Both magnetometer signals were measured with a simultaneous recording and processing of the differential signal. The realtime result of the subtraction of the reference channel is demonstrated in Fig. 3. A line-frequency synchronous filter combined with a bandpass filter suppressed the residues of $50 \mathrm{~Hz}$ interference with its harmonics. The measurement frequency bandwidth $(0.03-125) \mathrm{Hz}$ covered the most significant frequency range of the heart signal. The observed peak-to-peak amplitude of the noise band is about $500 \mathrm{fT}$. This corresponds to an effective resolution of $S_{f}^{1 / 2} \sim 17 \mathrm{fT} / \sqrt{ } \mathrm{Hz}$.

The stability of the system operation was demonstrated by the measurements of MCG maps. Similar to a standard 1-channel MCG mapping (see, e.g., Ref. 8), the magnetic field measurements were carried out at a $6 \times 6$ rectangular grid over the thorax with a distance of $4 \mathrm{~cm}$ between neighboring positions of the measurements in both directions (see Fig. 4). The total scan area was $20 \times 20 \mathrm{~cm}^{2}$. At each point the differential signal was averaged for $30 \mathrm{~s}$. The simultaneously measured electrocardiography channel served as a to AIP license or copyright, see http://apl.aip.org/apl/copyright.jsp 


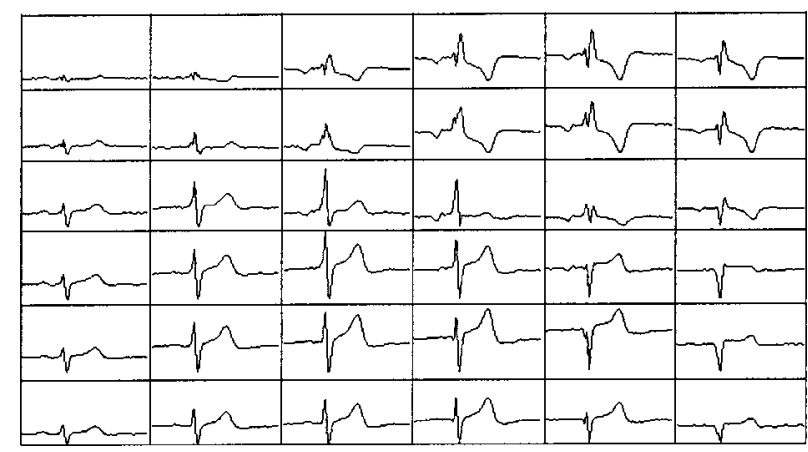

FIG. 4. The plots in the rectangular cells show the averaged MCG signals measured with the electronic gradiometer at $6 \times 6$ grid positions with a $4 \mathrm{~cm}$ pitch over the thorax. The recording time was $30 \mathrm{~s}$ per location.

trigger. The earlier-mentioned drift of the magnetic field in MSR of around $0.1 \mathrm{~Hz}$ and the effect of breathing caused some shifts of the averaged differential signal in Fig. 4.

Equi-inductional maps of magnetic field distribution were plotted on the basis of a two-dimensional interpolation according to the minimum curvature surface method (see Fig. 5). The MCG measurements for one mapping took about $30 \mathrm{~min}$. The magnetometers demonstrated a stable operation with no changes in the noise characteristics during the measurements. This allowed low noise two-dimensional plots of the magnetic field distribution to be obtained. The characteristics of the magnetometers were not changed even after a year of storage and multiple tests accompanied by thermal cyclings.

The demand to improve the parameters of HTS Josephson junctions has determined the present trend of reducing of the junction width to a submicrometer scale. In this case the main difficulty is how to maintain the superconducting pa-
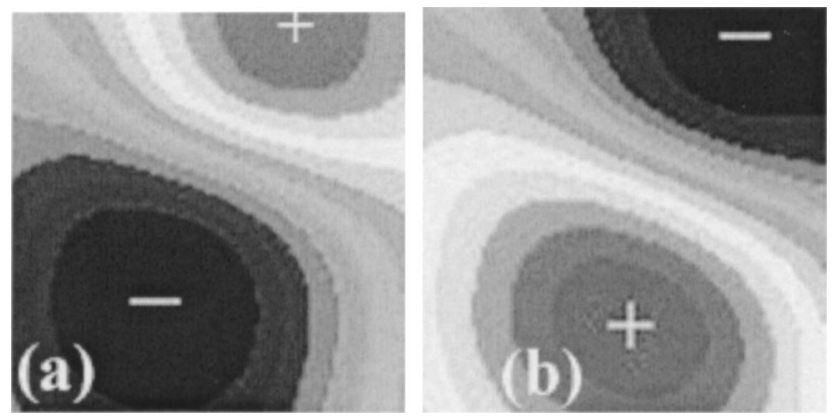

FIG. 5. Magnetic field distribution over the scan area at the moments of the (a) $S$ and (b) $T$ peaks, calculated on the basis of a two-dimensional interpolation. rameters at $77.4 \mathrm{~K}$ in narrow bicrystal junctions preventing degradation during the patterning process. Possible reasons for the degradation are chemical reactions in a humid atmosphere and deoxygenation of the $\mathrm{YBa}_{2} \mathrm{Cu}_{3} \mathrm{O}_{7-x}$ films. Both occur about 100 times faster along the bicrystal boundary compared to single crystalline films in the $c$-axis direction. It is important to achieve the highest possible microstructural quality of the $\mathrm{YBa}_{2} \mathrm{Cu}_{3} \mathrm{O}_{7-x}$ film and to keep the substrate cold during the ion beam etching process. A subsequent encapsulation then ensures the long-term stability of the sensor. The average size of the growth spirals of the $\mathrm{YBa}_{2} \mathrm{Cu}_{3} \mathrm{O}_{7-x}$ films, deposited by the high-oxygen-pressure dc-sputtering technique, ${ }^{6}$ is about $1 \mu \mathrm{m}$ compared to the about $0.3 \mu \mathrm{m}$ grains of conventional laser-ablated films. ${ }^{9}$ This makes it possible for the sputtered films to produce junctions between the individual growth spirals, thus contributing to the homogeneity and increasing the tunneling part of the critical current density of the junction. ${ }^{10} \mathrm{~A}$ detailed study of the transport and microstructural properties of submicrometer-wide bicrystal Josephson junctions prepared with the sputtered $\mathrm{YBa}_{2} \mathrm{Cu}_{3} \mathrm{O}_{7-x}$ films is in progress.

In conclusion, we investigated HTS dc-SQUID flip-chip magnetometers with submicrometer-wide bicrystal Josephson junctions. The overall resolution and stability of the flipchip magnetometers was demonstrated by operation at 77.4 $\mathrm{K}$ in a biomagnetic measurement system. The characteristics of the system correspond to demands for routine MCG measurements under clinical conditions in a standard magnetic shielded room.

${ }^{1}$ E. Dantsker, F. Ludwig, R. Kleiner, J. Clarke, M. Teepe, L. P. Lee, N. McN. Alford, and T. Button, Appl. Phys. Lett. 67, 725 (1995).

${ }^{2}$ D. Drung, F. Ludwig, W. Müller, U. Steinhoff, L. Trahms, H. Koch, Y. Q. Shen, M. B. Jensen, P. Vase, T. Holst, T. Freltoft, and G. Curio, Appl. Phys. Lett. 68, 1421 (1996).

${ }^{3}$ M. I. Faley, U. Poppe, K. Urban, D. N. Paulson, T. Starr, and R. L. Fagaly, IEEE Trans. Appl. Supercond. 11, 1383 (2001).

${ }^{4}$ R. Gross, P. Chaudhari, M. Kawasaki, and A. Gupta, Phys. Rev. B 42, 10735 (1990).

${ }^{5}$ H. Hilgenkamp and J. Mannhart, Appl. Phys. Lett. 73, 265 (1998).

${ }^{6}$ U. Poppe, N. Klein, U. Dähne, H. Soltner, C. L. Jia, B. Kabius, K. Urban, A. Lubig, K. Schmidt, S. Hensen, S. Orbach, G. Müller, and H. Piel, J. Appl. Phys. 71, 5572 (1992).

${ }^{7}$ M. I. Faley, U. Poppe, H. Soltner, C. L. Jia, M. Siegel, and K. Urban, Appl. Phys. Lett. 63, 2138 (1993).

${ }^{8}$ A. Gapelyuk, C. A. Copetti, A. Schirdewan, H. Schutt, M. Wiedemann, U. Meyerfeldt, M. A. Primin, Yu. V. Maslenikov, in: Biomag 96: Proceedings of the Tenth International Conference on Biomagnetism, edited by C. J. Aine, Y. Okada, G. Stroink, S. J. Swithenby, and C. C. Wood (Springer, New York, 2000); p. 464.

${ }^{9}$ B. Dam, N. J. Koeman, J. H. Rector, B. Stäuble-Pümpin, U. Poppe, and R. Griessen, Physica C 261, 1 (1996).

${ }^{10}$ E. Sarnelli and G. Testa, Physica C 371, 10 (2002). 